Article

\title{
Direct 2,3-O-Isopropylidenation of $\alpha$-D-Mannopyranosides and the Preparation of 3,6-Branched Mannose Trisaccharides
}

\author{
Rui Jiang, Guanghui Zong, Xiaomei Liang, Shuhui Jin, Jianjun Zhang * and Daoquan Wang \\ Department of Applied Chemistry, China Agricultural University, Beijing 100193, China \\ * Author to whom correspondence should be addressed; E-Mail: zhangjianjun@cau.edu.cn; \\ Tel.: +86-10-6273-2219.
}

Received: 31 March 2014; in revised form: 9 May 2014 / Accepted: 19 May 2014 /

Published: 22 May 2014

\begin{abstract}
A highly efficient, regioselective method for the direct 2,3-O-isopropylidenation of $\alpha$-D-mannopyranosides is reported. Treatment of various $\alpha$-D-mannopyranosides with 0.12 equiv of the $\mathrm{TsOH} \cdot \mathrm{H}_{2} \mathrm{O}$ and 2-methoxypropene at $70{ }^{\circ} \mathrm{C}$ gave $2,3-\mathrm{O}$-isopropylidene$\alpha$-D-mannopyranosides directly in $80 \% \sim 90 \%$ yields. Based on this method, a 3,6-branched $\alpha$-D-mannosyl trisaccharide was prepared in 50.4\% total yield using $p$-nitrophenyl 2,3-Oisopropylidene- $\alpha$-D-mannopyranoside as the starting material.
\end{abstract}

Keywords: 2,3-O-isopropylidenation; $\alpha$-D-mannosides; trisaccharide

\section{Introduction}

Many carbohydrates isolated from natural products were found to be involved in a wide range of biological processes. Further investigation of the structure-activity relationships of these carbohydrates normally is often restricted, since the isolated compounds can't meet the purity and quantity needs of pharmacologists. Thus, the synthesis of structurally complex carbohydrates via chemical methods has become very important, and various kinds of suitably functionalized monosaccharide building blocks are basic requirements in the synthesis of oligosaccharides [1-4].

The isopropylidene moiety is one of the frequently used protecting groups in oligosaccharide synthesis for the temporary protection of hydroxyl groups. 2,3-O-Isopropylidene- $\alpha$-D-mannopyranosides are important building blocks in the synthesis of mannose-containing derivatives [5-7], and many methods have been reported for the preparation of these compounds. In 1977, Evans [8] disclosed that by the reaction of methyl $\alpha$-D-mannopyranoside with 2,2-dimethoxypropane in the 
presence of sulfuric acid for $48 \mathrm{~h}$, methyl 2,3-O-isopropylidene- $\alpha$-D-mannopyranoside was obtained in $56 \%$ yield. Obviously, this reaction is time-consuming and the yield is only barely acceptable. In most cases, 2,3-O-isopropylidene- $\alpha$-D-mannopyranosides are prepared in two steps from $\alpha$-D-manno- pyranosides: 2,3:4,6-di- $O$-isopropylidenation of mannopyranosides followed by selectively removal of 4,6-O-isopropylidene groups in the presence of acids. The reported acids including aq. $\mathrm{HCl}$ [9], $60 \%$ aq. $\mathrm{AcOH}$ [10], aq. $\mathrm{H}_{2} \mathrm{SO}_{4}$ [11], $\mathrm{Zn}\left(\mathrm{NO}_{3}\right)_{2} \cdot 6 \mathrm{H}_{2} \mathrm{O}$ [12], $\mathrm{BiCl}_{3}$ [13]. To better control the selectivity and efficiency of the hydrolysis, Dowex $\mathrm{H}^{+}$ion-exchange resin [14], $\mathrm{FeCl}_{3} \cdot 6 \mathrm{H}_{2} \mathrm{O}$ on silica [15], $\mathrm{NaHSO}_{4}$ on silica [16] and $\mathrm{HClO}_{4}$ on silica [17] were also used in some cases. Nevertheless, many of these methods suffer from the use of corrosive materials, rigorous reaction conditions and incompatibility with various other protecting groups. Some of the acids used in the reaction are strongly acidic and the selectivity was compromised, leading to decreased yields of the desired products. Here we wish to report a direct and regioselective 2,3- $O$-isopropylidenation of $\alpha$-D-mannopyranosides method, and its application in the synthesis of mannose oligosaccharide exemplified by the efficient preparation of a 3,6-branched $\alpha$-D-mannosyl trisaccharide.

\section{Results and Discussion}

As shown in Scheme 1, reaction of the known $p$-methoxyphenyl $\alpha$-D-mannopyranoside [18] (1a, $1 \mathrm{mmol})$ with 2-methoxypropene $(1.05 \mathrm{mmol})$ in anhydrous $N, N$-dimethylformamide containing a trace of $p$-toluenesulfonic acid $(0.02 \mathrm{mmol})$ at room temperature occurs preferentially at the primary hydroxyl group, to give $p$-methoxyphenyl 4,6-O-isopropylidene- $\alpha$-D-mannopyranoside (2a) [19]. The reaction was terminated at the end of the reaction $(2 \mathrm{~h})$ by addition of triethylamine to neutralize the $p$-toluene-sulfonic acid. Incidentally, we discovered that after extension of the reaction time to $10 \mathrm{~h}$ or more without neutralizing the $p$-toluenesulfonic acid, a new by-product 3a was formed in $20 \% \sim 30 \%$ yields. The by-product 3a was purified and it was confirmed to be $p$-methoxyphenyl 2,3-O-isopropylidene- $\alpha$-Dmannopyranoside, judging from its ${ }^{1} \mathrm{H}-\mathrm{NMR}$ and ${ }^{13} \mathrm{C}-\mathrm{NMR}$ spectrum identical to the published ones [5]. Obviously, rearrangement of the $p$-methoxyphenyl 4,6- $O$-isopropylidene- $\alpha$-D-mannopyranoside to $p$-methoxyphenyl 2,3-O-isopropylidene- $\alpha$-D-mannopyranoside took place during the course of the reaction. It is important to note that, after a series of experiments, the efficiency of the rearrangement was found to depend on the amount of acid added and the reaction temperature, and the isolated yields of $\mathbf{3 a}$ were greatly improved under the optimized reaction conditions, and finally, reaction of compound 1a with 2-methoxypropene (1.05 equiv) in anhydrous $N, N$-dimethylformamide containing 0.1 equiv of p-toluene-sulfonic acid at $70{ }^{\circ} \mathrm{C}$ for $4 \mathrm{~h}$, gave $3 \mathrm{a}$ in $93 \%$ yield.

Scheme 1. Synthesis of $\mathbf{2 a}$ and $\mathbf{3 a}$.

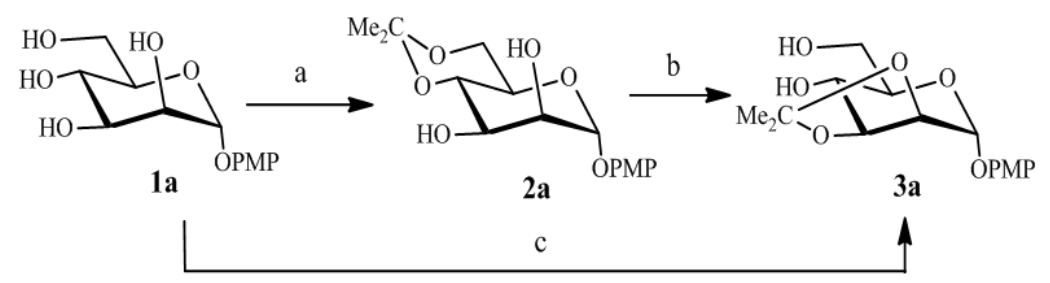

Reagents and Conditions: (a) 2-methoxypropene, TsOH $\cdot \mathrm{H}_{2} \mathrm{O}$, DMF, r.t., 2 h, 94\%; (b) r.t., 10 h, $28 \%$; (c) 2-methoxypropene, $\mathrm{TsOH} \cdot \mathrm{H}_{2} \mathrm{O}, \mathrm{DMF}$, r.t. then $70{ }^{\circ} \mathrm{C}, 4 \mathrm{~h}, 93 \%$. 
A number of suitably functionalized mannose intermediates 1b [20], 1c [6], 1d [7], 1e [21], 1f [22] and 1g [23] were prepared from the commercially available D-mannose, using earlier reported reaction conditions. With the optimized reaction conditions in hand, $\alpha$-D-mannopyranosides 1b 1f were used as substrates to investigate the general applicability of the method for the direct synthesis of 2,3-O-isopropylidene- $\alpha$-D-mannopyranosides $\mathbf{3 b} \sim \mathbf{3 f}$. Though the reaction conditions are slightly different for each substrate, we were delighted to find that good to excellent yields $(85 \% \sim 93 \%)$ of the corresponding 2,3-O-isopropylidene- $\alpha$-D-mannopyranoside derivatives were obtained in all cases, and the structure were confirmed by their NMR data (Table 1).

Table 1. Synthesis of 2,3-O-isopropylidene- $\alpha$-D-mannopyranosides derivatives.

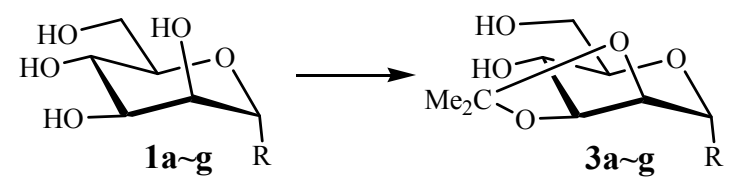

\begin{tabular}{|c|c|c|c|c|c|c|c|}
\hline Entry & Reaction & $\mathbf{R}$ & TsOH (equiv) & $\mathbf{T}\left({ }^{\circ} \mathrm{C}\right)$ & Time(h) & Yield (\%) & Ref. * \\
\hline 1 & $1 a \longrightarrow 3 a$ & $\mathrm{O}-10 \mathrm{OMe}$ & 0.1 & 70 & 4 & 93 & [5] \\
\hline 2 & $1 \mathrm{~b} \longrightarrow 3 \mathrm{~b}$ & $s-<$ & 0.3 & 50 & 1.5 & 89 & - \\
\hline 3 & $1 \mathrm{c} \longrightarrow 3 \mathrm{c}$ & $\mathrm{O}\urcorner=$ & 0.1 & 70 & 2 & 91 & {$[6]$} \\
\hline 4 & $1 d \longrightarrow 3 d$ & & 0.3 & 70 & 3.5 & 92 & [7] \\
\hline 5 & $1 \mathrm{e} \longrightarrow 3 \mathrm{e}$ & s- & 0.5 & 50 & 4.5 & 85 & [21] \\
\hline 6 & $1 \mathrm{f} \longrightarrow 3 \mathrm{f}$ & s-田 & 0.1 & 70 & 2 & 88 & [22] \\
\hline 7 & $1 \mathrm{~g} \longrightarrow 3 \mathrm{~g}$ & $\mathrm{O}-(-1) \mathrm{NO}_{2}$ & 0.3 & 70 & 2 & 90 & [23] \\
\hline
\end{tabular}

* Refs. of known products.

The above synthesized 2,3- $O$-isopropylidene- $\alpha$-D-mannopyranosides are useful building blocks for the preparation of mannose-containing oligosaccharides and glycoconjugates. As an example, a 3,6-branched mannose trisaccharide (10, Scheme 2), the core structure of $N$-linked glycan mannose oligosaccharides, was synthesized efficiently. $N$-linked glycan mannose oligosaccharides play a vital role in fundamental biological processes including cell differentiation, malignant transformation, human CD2 adhesion function and HIV infection, and the 3,6-branched mannosyl trisaccharide is reported to be the much better ligand of mannose-specific binding proteins than mono- and linear oligomannosides [24-27]. Due to their biological importance, 3,6-branched mannosides have become the synthetic focus of many research groups [28-31] and the development of new synthetic strategies for their efficient construction is of continue interest.

Several synthetic methods have already been reported for 3,6-branched mannose trisaccharides [32-41]. In 1991, Kaur and Hindsgaul [34] revealed a fifteen-step method for the synthesis of 3,6-branched trisaccharide from commercial available starting materials, but many of these steps involved time-consuming and delicate protecting group manipulations to synthesize 2,4-diprotected mannosides for the subsequent glycosylation. Later, Hindsgaul [35] and Bencomo [37] reported a general approach based on random glycosylation for octyl- and 5-azido-3-oxapentyl- $\alpha-D-$ mannopyranosides, respectively. They used acetobromosugars as the glycosyl donor and mercury (II) cyanide and mercury (III) bromide as the catalysts. In 2000, Kobayashi [38] examined a similar but more convenient glycosylation of $p$-nitrophenyl a-D-mannopyranoside using per- $O$-acetyl-a-D- 
mannopyranosyl imidate as the donor. The reaction gave a desired 3,6-branched trisaccharide in $42 \%$ yield as the major product, together with di-, tri- and tetrasaccharide byproducts. Orthoester chemistry is an efficient method for the synthesis of these 3,6-branched trisaccharides [32,36,40-42]. Arnarp and Lonngren [42] described in 1978 a slightly more circuitous route that starts with stannylated benzyl a-D-mannopyranoside, the 3,6-branched mannotrioside was obtained in good yield via a protection-deprotection sequence that circumvents the orthoester rearrangement. In 1993, Oscarson [36], and in 2005, Backinowsky [40] and Mukhopadhyay [41] used orthoester chemistry to provide direct access to a 2,4-diprotected mannoside acceptor for the subsequent glycosylation with a mannoside donor. This approach was more convenient and efficient and the desired 3,6-branched mannotrioside was obtained in satisfactory yield. In this paper we describe a linear synthesis of the target 3,6-branched mannotrioside (10) using $p$-nitrophenyl 2,3- $O$-isopropylidene- $\alpha$-D-mannopyranoside (3g) as the mannoside acceptor and 2,3,4,6-tetra- $O$-benzoyl- $\alpha$-D-mannopyranoside trichloroacetimidate (4) as the donor. The 3,6-branched $\alpha$-D-mannosyl trisaccharide (10) was obtained in $50.4 \%$ total yield (Scheme 2).

Scheme 2. Synthesis of 3,6-branched $\alpha$-D-mannose trisaccharide $\mathbf{1 0}$.

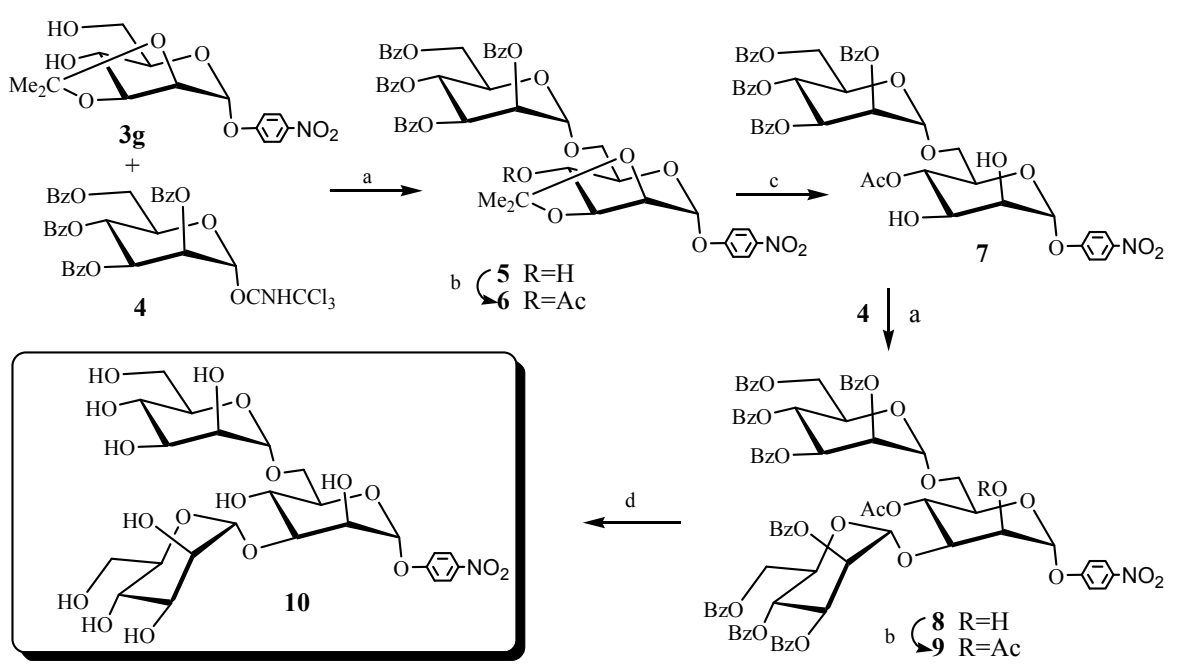

Reagents and conditions: (a) TMSOTf, $\mathrm{CH}_{2} \mathrm{Cl}_{2},-15{ }^{\circ} \mathrm{C}$ to rt, $2 \mathrm{~h}, 92 \%$ for 5, $86 \%$ for $\mathbf{8}$; (b) $\mathrm{Py}, \mathrm{Ac}_{2} \mathrm{O}, 96 \%$ for 6, $95 \%$ for 9 ; (c) $70 \% \mathrm{AcOH}, 70{ }^{\circ} \mathrm{C}, 2 \mathrm{~h}, 91 \%$; (d) satd $\mathrm{NH}_{3}-\mathrm{MeOH}, \mathrm{rt}, 120 \mathrm{~h}, 73 \%$.

\section{Experimental Section}

\subsection{General Methods}

Optical rotations were determined with a Perkin-Elmer model 241-MC automatic polarimeter for soln in a 1-dm, jacketed cell. ${ }^{1} \mathrm{H}$ and ${ }^{13} \mathrm{C}-\mathrm{NMR}$ spectra were recorded with Bruker DPX300 and Bruker AVANCE600 spectrometers in $\mathrm{CDCl}_{3}$ or $\mathrm{D}_{2} \mathrm{O}$ solns. Internal references: TMS $\left(\delta 0.000 \mathrm{ppm}\right.$ for $\left.{ }^{1} \mathrm{H}\right)$, $\mathrm{CDCl}_{3}\left(\delta 77.00 \mathrm{ppm}\right.$ for ${ }^{13} \mathrm{C}$ ), HOD $\left(\delta 4.700\right.$ for ${ }^{1} \mathrm{H}$ ). High-resolution mass spectra (HRMS) was performed by the Peking University. Thin-layer chromatography (TLC) was performed on silica gel $\mathrm{HF}$ with detection by charring with $30 \%(\mathrm{v} / \mathrm{v}) \mathrm{H}_{2} \mathrm{SO}_{4}$ in $\mathrm{MeOH}$ or by UV detection. Column chromatography was conducted by elution of a column of silica gel (200-300 mesh) with 
EtOAc/petroleum ether (bp 60-90 ${ }^{\circ} \mathrm{C}$ ) as the eluent. Solns were concd at a temperature $<60{ }^{\circ} \mathrm{C}$ under diminished pressure.

\subsection{Chemical Synthesis: Representative Procedure for the Synthesis of 2,3-O-Isopropylidene- $\alpha-D-$} mannopyranosides $\mathbf{3 a} \sim \mathbf{3 f}$

To a solution of compound $\mathbf{1 a} \sim \mathbf{1 g}(2 \mathrm{mmol})$ in anhydrous DMF $(20 \mathrm{~mL})$ was added $\mathrm{TsOH} \cdot \mathrm{H}_{2} \mathrm{O}$ $(0.2 \sim 1 \mathrm{mmol})$ and 2-methoxypropene $(0.2 \mathrm{~mL}, 2.1 \mathrm{mmol})$ under $\mathrm{N}_{2}$ atmosphere. The mixture was stirred at $\mathrm{rt}$ for $1 \mathrm{~h}$ and then for another $1 \sim 4 \mathrm{~h}$ at $50 \sim 70{ }^{\circ} \mathrm{C}$, at the end of which time TLC (EtOAc) indicated that the reaction was complete. The reaction mixture was neutralized with $\mathrm{Et}_{3} \mathrm{~N}$, and then concentrated under reduced pressure to remove DMF, the residue was dissolved in $\mathrm{CH}_{2} \mathrm{Cl}_{2}(50 \mathrm{~mL})$, and washed with water $(20 \mathrm{~mL})$, then the organic phase was dried over $\mathrm{Na}_{2} \mathrm{SO}_{4}$. Evaporation and purification by flash column chromatography afforded compounds $\mathbf{3 a} \sim \mathbf{3 g}$.

p-Methoxyphenyl 2,3-O-isopropylidene- $\alpha$-D-mannopyranoside (3a): $(0.6 \mathrm{~g}, 93 \%)$. $[\alpha]_{D}^{25}+69.7^{\circ}(c 1.0$ $\left.\mathrm{CHCl}_{3}\right) .{ }^{1} \mathrm{H}-\mathrm{NMR}\left(300 \mathrm{MHz}, \mathrm{CDCl}_{3}\right) \delta 7.00-6.82(2 \mathrm{~m}, 4 \mathrm{H}, \mathrm{Ar}-H), 5.67(\mathrm{~s}, 1 \mathrm{H}, H-1), 4.38(\mathrm{~d}$, $\left.J_{2,3}=5.7 \mathrm{~Hz}, 1 \mathrm{H}, H-2\right), 4.32(\mathrm{~m}, 1 \mathrm{H}, H-3), 3.84-3.78\left(\mathrm{~m}, 7 \mathrm{H}, H-4, H-5, H-6, O C H_{3}\right), 2.82(\mathrm{~d}, J=3.9 \mathrm{~Hz}$, $1 \mathrm{H}, 4-\mathrm{OH}), 2.04(\mathrm{~m}, 1 \mathrm{H}, 6-\mathrm{OH}), 1.56,1.41\left(2 \mathrm{~s}, 6 \mathrm{H}, \mathrm{Me}_{2} \mathrm{C}\right) .{ }^{13} \mathrm{C}-\mathrm{NMR}\left(75 \mathrm{MHz}, \mathrm{CDCl}_{3}\right): \delta 155.1$, 149.7, 117.8, 114.6, 109.8, 96.4, 78.5, 75.6, 70.2, 69.0, 61.8, 55.5, 27.9, 26.1. HRMS for $\mathrm{C}_{16} \mathrm{H}_{26} \mathrm{NO}_{7}$ $\left(\mathrm{M}+\mathrm{NH}_{4}\right)^{+}$344.17038. Found: 344.17035 .

Isopropylthio 2,3-O-isopropylidene- $\alpha$-D-mannopyranoside (3b): $(0.49 \mathrm{~g}, 89 \%) \cdot[\alpha]_{D}^{25}+128.7^{\circ}(c) 1.0$ $\left.\mathrm{CHCl}_{3}\right) .{ }^{1} \mathrm{H}-\mathrm{NMR}\left(300 \mathrm{MHz}, \mathrm{CDCl}_{3}\right) \delta 5.63(\mathrm{~s}, 1 \mathrm{H}, H-1), 4.18\left(\mathrm{~d}, J_{2,3}=5.5 \mathrm{~Hz}, 1 \mathrm{H}, H-2\right), 4.11$ (m, $1 \mathrm{H}, H-3), 4.01(\mathrm{~m}, 1 \mathrm{H}, H-5), 3.88-3.77$ (m, 3H, H-4,H-6), 3.10 (m, 1H, SCH(CH3)2), 2.89 (d, $J=4.0 \mathrm{~Hz}, 1 \mathrm{H}, \mathrm{OH}), 2.16(\mathrm{~m}, 1 \mathrm{H}, \mathrm{OH}), 1.54,1.36(2 \mathrm{~s}, 6 \mathrm{H}, M e 2 \mathrm{C}) .1 .35(\mathrm{~d}, J=4.8 \mathrm{~Hz}, 3 \mathrm{H}$, $\mathrm{CH}(\mathrm{CH} 3) 2), 1.30(\mathrm{~d}, J=6.9 \mathrm{~Hz}, 3 \mathrm{H}, \mathrm{CH}(\mathrm{CH} 3) 2)$. HRMS for $\mathrm{C}_{12} \mathrm{H}_{26} \mathrm{SNO}_{5}\left(\mathrm{M}+\mathrm{NH}_{4}\right)^{+} 296.15262$. Found: 296.15259.

Allyl 2,3-O-isopropylidene- $\alpha$-D-mannopyranoside $(3 \mathrm{c})$ : $(0.47 \mathrm{~g}, 91 \%)$. $[\alpha]_{D}^{25}+68.2^{\circ}\left(c 1.0 \mathrm{CHCl}_{3}\right)$. ${ }^{1} \mathrm{H}-\mathrm{NMR}\left(300 \mathrm{MHz}, \mathrm{CDCl}_{3}\right) \delta$ 5.96-5.85 (m, 1H, $\left.\mathrm{OCH}_{2} \mathrm{CHCH}_{2}\right), 5.33-5.19\left(\mathrm{~m}, 2 \mathrm{H}, \mathrm{CH}_{2} \mathrm{CHCH}_{2} \mathrm{O}\right)$, $4.89\left(\mathrm{~d}, J_{1,2}=1.2 \mathrm{~Hz}, 1 \mathrm{H}, H-1\right), 4.21-3.68$ (m, 8H, H-2, H-3, H-4, H-5, H-6, $\mathrm{CH}_{2} \mathrm{CHCH}_{2} \mathrm{O}$ ), 2.63-2.57 $(\mathrm{m}, 2 \mathrm{H}, 2 \mathrm{OH}), 1.53,1.43\left(2 \mathrm{~s}, 6 \mathrm{H}, \mathrm{Me}_{2} \mathrm{C}\right)$. HRMS for $\mathrm{C}_{12} \mathrm{H}_{21} \mathrm{O}_{6}(\mathrm{M}+\mathrm{H})^{+} 261.13326$. Found: 261.13321.

Benzyl 2,3-O-isopropylidene- $\alpha$-D-mannopyranoside (3d): $(0.57 \mathrm{~g}, 92 \%)$. $[\alpha]_{D}^{25}+77.0^{\circ}\left(c 1.0 \mathrm{CHCl}_{3}\right)$. ${ }^{1} \mathrm{H}-\mathrm{NMR}\left(300 \mathrm{MHz}, \mathrm{CDCl}_{3}\right) \delta 7.40-7.26(\mathrm{~m}, 5 \mathrm{H}, \mathrm{ArH}), 5.12(\mathrm{~s}, 1 \mathrm{H}, H-1), 4.74(\mathrm{~d}, J=11.7 \mathrm{~Hz}, 1 \mathrm{H}$, $\left.\mathrm{OCH}_{2} \mathrm{Ph}\right), 4.54\left(\mathrm{~d}, J=11.7 \mathrm{~Hz}, 1 \mathrm{H}, \mathrm{OCH}_{2} \mathrm{Ph}\right), 4.20-4.16(\mathrm{~m}, 2 \mathrm{H}), 3.85-3.83(\mathrm{~m}, 2 \mathrm{H}), 3.78-3.66(\mathrm{~m}$, $2 \mathrm{H}), 3.30-2.88(\mathrm{~s}, 1 \mathrm{H}, \mathrm{OH}), 2.35(\mathrm{~s}, 1 \mathrm{H}, \mathrm{OH}), 1.52,1.34\left(2 \mathrm{~s}, 6 \mathrm{H}, M e_{2} C\right)$. HRMS for $\mathrm{C}_{16} \mathrm{H}_{26} \mathrm{NO}_{6}$ $\left(\mathrm{M}+\mathrm{NH}_{4}\right)^{+}$328.17546. Found: 328.17548 .

Ethyl 2,3-O-isopropylidene-1-thio- $\alpha$-D-mannopyranoside (3e): $(0.44 \mathrm{~g}, 85 \%) .[\alpha]_{D}^{25}+136.8^{\circ}(c 1.0$ $\mathrm{CHCl}_{3}$ ). ${ }^{1} \mathrm{H}-\mathrm{NMR}\left(300 \mathrm{MHz}, \mathrm{CDCl}_{3}\right) \delta 5.58(\mathrm{~s}, 1 \mathrm{H}, H-1), 4.19(\mathrm{~m}, 1 \mathrm{H}, H-2), 4.14(\mathrm{~m}, 1 \mathrm{H}, H-3)$, 3.99-3.93 (m, 1H, H-5), 3.88-3.77 (m, 3H, H-4, H-6), 2.74-2.49 (m, 3H, SCH2, OH), 2.07 (t, $J=6.5 \mathrm{~Hz}$, $1 \mathrm{H}, \mathrm{OH}), 1.54\left(\mathrm{~s}, 3 \mathrm{H}, \mathrm{Me}_{2} \mathrm{C}\right), 1.40-1.25\left(\mathrm{~m}, 6 \mathrm{H}, \mathrm{SCH}_{2} \mathrm{CH}_{3}, M e_{2} C\right.$ ). HRMS for $\mathrm{C}_{11} \mathrm{H}_{24} \mathrm{SNO}_{5}\left(\mathrm{M}+\mathrm{NH}_{4}\right)^{+}$ 282.13697. Found: 282.13696. 
Phenyl 2,3-O-isopropylidene-1-thio- $\alpha$-D-mannopyranoside (3f): $(0.55 \mathrm{~g}, 88 \%)$. $[\alpha]_{D}^{25}+197.8^{\circ}(c) 1.0$ $\mathrm{CHCl}_{3}$ ). ${ }^{1} \mathrm{H}-\mathrm{NMR}\left(300 \mathrm{MHz}, \mathrm{CDCl}_{3}\right) \delta$ 7.50-7.26 (m, 5H, $\left.\mathrm{ArH}\right), 5.81(\mathrm{~s}, 1 \mathrm{H}, H-1), 4.35\left(\mathrm{~d}, J_{2,3}=5.5 \mathrm{~Hz}\right.$, $1 \mathrm{H}, H-2), 4.18\left(\mathrm{dd}, J_{2,3}=5.5 \mathrm{~Hz}, J_{3,4}=7.5 \mathrm{~Hz}, 1 \mathrm{H}, H-3\right), 4.08-4.02(\mathrm{~m}, 1 \mathrm{H}, H-5), 3.83-3.70$ (m, $H-4$, $H-6), 2.92(\mathrm{~d}, J=4.0 \mathrm{~Hz}, \mathrm{OH}), 1.95(\mathrm{t}, 1 \mathrm{H}, J=6.3 \mathrm{~Hz}, \mathrm{OH}), 1.54,1.38\left(2 \mathrm{~s}, 6 \mathrm{H}, \mathrm{Me}_{2} \mathrm{C}\right)$. HRMS for $\mathrm{C}_{15} \mathrm{H}_{24} \mathrm{SNO}_{5}\left(\mathrm{M}+\mathrm{NH}_{4}\right)^{+}$330.13697. Found: 330.13699 .

p-Nitrophenyl 2,3-O-isopropylidene- $\alpha$-D-mannopyranoside $(\mathbf{3 g}):(0.61 \mathrm{~g}, 90 \%)$. $[\alpha]_{0}^{25}+94.8^{\circ}(c) 1.0$ $\mathrm{CHCl}_{3}$ ). ${ }^{1} \mathrm{H}-\mathrm{NMR}(300 \mathrm{MHz}, \mathrm{CDCl} 3) \delta \mathrm{ppm} 8.25-8.20(\mathrm{~m}, 2 \mathrm{H}, \mathrm{Ar}-H), 7.18-7.13(\mathrm{~m}, 2 \mathrm{H}, \mathrm{Ar}-H), 5.90$ $(\mathrm{s}, 1 \mathrm{H}, \mathrm{H}-1), 4.38\left(\mathrm{~d}, J_{2,3}=5.7 \mathrm{~Hz}, 1 \mathrm{H}, H-2\right), 4.32\left(\mathrm{dd}, J_{2,3}=5.7 \mathrm{~Hz}, J_{3,4}=7.3 \mathrm{~Hz}, 1 \mathrm{H}, H-3\right), 3.88-3.78$ (m, 3H, H-4, $2 \times \mathrm{H}-6), 3.67-3.62$ (m, 1H, H-5), 2.96 (d, 1H, J=4.0 Hz, OH), 2.05 (d, 1H, J=3.6 Hz, $\mathrm{OH}), 1.58,1.42\left(2 \mathrm{~s}, 6 \mathrm{H}, 2 \times \mathrm{C}-\mathrm{CH}_{3}\right)$. HRMS for $\mathrm{C}_{15} \mathrm{H}_{23} \mathrm{NO}_{8}\left(\mathrm{M}+\mathrm{NH}_{4}\right)^{+}$359.1449. Found: 359.14481.

\section{4-Nitrophenyl 2,3,4,6-tetra-O-benzoyl- $\alpha$-D-mannopyranosyl-( $1 \rightarrow 6)$-2,3-O-isopropylidene- $\alpha-D$ -} mannopyranoside (5): To a cooled $\left(-15^{\circ} \mathrm{C}\right)$ solution of $3 \mathrm{~g}(1.5 \mathrm{~g}, 4.4 \mathrm{mmol})$ and $4(3.4 \mathrm{~g}, 4.6 \mathrm{mmol})$ in anhydrous, redistilled $\mathrm{CH}_{2} \mathrm{Cl}_{2}(80 \mathrm{~mL})$ was added $4 \AA$ molecular sieves $(2 \mathrm{~g})$ and the mixture was stirred under a $\mathrm{N}_{2}$ atmosphere for $30 \mathrm{~min}$. Then TMSOTf $(16 \mu \mathrm{L} 0.09 \mathrm{mmol}$, diluted with $10 \mathrm{~mL}$ redistilled $\mathrm{CH}_{2} \mathrm{Cl}_{2}$ ) was added to the mixture dropwise. The reaction mixture was stirred for another $2 \mathrm{~h}$, during which time the mixture was allowed to gradually warm to ambient temperature. TLC (petroleum ether-EtOAc 2:1) indicated that the reaction was complete. Then the reaction mixture was quenched with $\mathrm{Et}_{3} \mathrm{~N}$ (2 drops) and filtrated. The filtrate was evaporated in vacuo to give a residue, which was purified by silica gel column chromatography (petroleum ether-EtOAc 3:1) to give disaccharide 5 $(3.7 \mathrm{~g}, 92 \%)$ as a white foam. $[\alpha]_{D}^{25}-62.6^{\circ}\left(c 0.5 \mathrm{CHCl}_{3}\right) .{ }^{1} \mathrm{H}-\mathrm{NMR}\left(300 \mathrm{MHz}, \mathrm{CDCl}_{3}\right): \delta 8.31-7.23(\mathrm{~m}$, 24H, Bz- $H, \operatorname{Ar}-H$ ), 6.07 (t, $J_{3,4}=J_{4,5}=10.0 \mathrm{~Hz}, 1 \mathrm{H}, \mathrm{H}-4$ '), 5.94 (s, $\left.1 \mathrm{H}, \mathrm{H}-1^{\prime}\right), 5.74$ (dd, $J_{2,3}=3.3 \mathrm{~Hz}$, $\left.J_{3,4}=10.0 \mathrm{~Hz}, 1 \mathrm{H}, \mathrm{H}-3^{\prime}\right), 5.56\left(\mathrm{dd}, J_{1,2}=1.7 \mathrm{~Hz}, J_{2,3}=3.3 \mathrm{~Hz}, 1 \mathrm{H}, \mathrm{H}-2^{\prime}\right), 5.10\left(\mathrm{~d}, 1 \mathrm{H}, J_{1,2}=1.7 \mathrm{~Hz}, \mathrm{H}-1\right)$, 4.74-4.68 (m, 1H), 4.50-4.41 (m, 3H), $4.34(\mathrm{~m}, 1 \mathrm{H}, \mathrm{H}-4), 4.01(\mathrm{~m}, 1 \mathrm{H}), 3.91-3.82(\mathrm{~m}, 3 \mathrm{H}), 2.68(\mathrm{~s}, 1 \mathrm{H}$, $\mathrm{OH}), 1.60,1.43\left(2 \mathrm{~s}, 6 \mathrm{H}, 2 \times \mathrm{C}-\mathrm{CH}_{3}\right) .{ }^{13} \mathrm{C}-\mathrm{NMR}\left(75 \mathrm{MHz}, \mathrm{CDCl}_{3}\right): \delta 166.2,165.6,165.2(2)(4 \times \mathrm{COPh})$, $160.6\left(\mathrm{CNO}_{2}\right), 142.9,133.4,133.1,133.0,129.8,129.7,129.6,129.2,129.0,128.9,128.5,128.4,128.3$, 128.2, 126.0, 116.2, 110.3, 97.6, 95.7 (2× C-1), 78.5, 75.3, 70.3, 70.0, 69.6, 69.2, 68.9, 67.1, 66.7, 62.9, 28.0, $26.3\left(2 \times \mathrm{C}-\mathrm{CH}_{3}\right)$. HRMS for $\mathrm{C}_{49} \mathrm{H}_{49} \mathrm{~N}_{2} \mathrm{O}_{17}\left(\mathrm{M}+\mathrm{NH}_{4}\right)^{+}$937.30257. Found: 937.30133.

4-Nitrophenyl 2,3,4,6-tetra-O-benzoyl- $\alpha$-D-mannopyranosyl-(1 $\rightarrow 6)$-4-O-acetyl-2,3-O-isopropylidene$\alpha$-D-mannopyranoside (6): To a solution of 5 (3.6 g, $4 \mathrm{mmol})$ in pyridine $(30 \mathrm{~mL})$ was added $\mathrm{Ac}_{2} \mathrm{O}$ $(3.7 \mathrm{~mL}, 40 \mathrm{mmol})$. The reaction mixture was stirred at $\mathrm{rt}$ for $12 \mathrm{~h}$, at the end of which time TLC (petroleum ether-EtOAc 2:1) indicated that the reaction was complete. The reaction mixture was concentrated, and then the residue was purified by flash column chromatography on a silica gel column (petroleum ether-EtOAc 3:1) to give compound $6(3.6 \mathrm{~g}, 96 \%)$ as a foamy solid. $[\alpha]_{D}^{25}-53.3^{\circ}$ (c $\left.0.3 \mathrm{CHCl}_{3}\right) .{ }^{1} \mathrm{H}-\mathrm{NMR}\left(300 \mathrm{MHz}, \mathrm{CDCl}_{3}\right): \delta 8.29$ (d, $\left.J=9.1 \mathrm{~Hz}, 2 \mathrm{H}, \mathrm{Bz}-H\right), 8.10,7.80(2 \mathrm{~d}, J=7.4 \mathrm{~Hz}$, $\left.4 \mathrm{H}, \mathrm{C}_{6} H_{4} \mathrm{NO}_{2}\right), 7.58-7.24(\mathrm{~m}, 14 \mathrm{H}, \mathrm{Bz}-H), 6.05$ (t, $J_{3,4}=J_{4,5}=10.0 \mathrm{~Hz}, 1 \mathrm{H}, \mathrm{H}-4$ '), $5.95\left(\mathrm{~s}, 1 \mathrm{H}, \mathrm{H}-1^{\prime}\right)$, $5.65\left(\mathrm{dd}, J_{2,3}=3.3 \mathrm{~Hz}, J_{3,4}=10.0 \mathrm{~Hz}, 1 \mathrm{H}, \mathrm{H}-3^{\prime}\right), 5.46\left(\mathrm{~m}, 1 \mathrm{H}, \mathrm{H}-2^{\prime}\right), 5.10\left(\mathrm{t}, J_{3,4}=J_{4,5}=10.3 \mathrm{~Hz}, 1 \mathrm{H}\right.$, H-4), 5.01 (s, 1H, H-1), 4.73 (d, $\left.J_{3,4}=10.3 \mathrm{~Hz}, 1 \mathrm{H}, \mathrm{H}-3\right), 4.49-4.40$ (m, 4H), 4.05-3.88 (m, 2H), 3.53 $(\mathrm{m}, 1 \mathrm{H}), 2.21(\mathrm{~s}, 3 \mathrm{H}, \mathrm{CH} / \mathrm{CO}), 1.60,1.42\left(2 \mathrm{~s}, 6 \mathrm{H}, 2 \times \mathrm{C}-\mathrm{CH}_{3}\right) .{ }^{13} \mathrm{C}-\mathrm{NMR}\left(75 \mathrm{MHz}, \mathrm{CDCl}_{3}\right): \delta 169.7$ $\left(\mathrm{COCH}_{3}\right), 166.1,165.6,165.2,165.1(4 \times \mathrm{COPh}), 160.4\left(\mathrm{CNO}_{2}\right), 143.1,133.4,133.1,133.0,129.8$, 
129.7, 129.6, 129.1, 129.0, 128.9, 128.5, 128.4, 128.2, 126.0, 116.1, 110.6, 97.3, $95.4(2 \times \mathrm{C}-1), 77.1$, $75.4,75.1,70.2,69.5,69.3,69.0,68.3,66.9,66.8,62.9,27.5,26.3\left(2 \times \mathrm{C}-\mathrm{CH}_{3}\right), 20.8\left(\mathrm{COCH}_{3}\right) . \mathrm{HRMS}$ for $\mathrm{C}_{51} \mathrm{H}_{51} \mathrm{~N}_{2} \mathrm{O}_{18}\left(\mathrm{M}+\mathrm{NH}_{4}\right)^{+}$979.31314. Found: 979.31201 .

4-Nitrophenyl 2,3,4,6-tetra-O-benzoyl- $\alpha$-D-mannopyranosyl-(1 $\rightarrow 6)$-4-O-acetyl- $\alpha$-D-mannopyranoside (7): Compound 6 (3.5 g, $3.6 \mathrm{mmol})$ was dissolved in $70 \% \mathrm{AcOH}(60 \mathrm{~mL})$ and stirred for $2 \mathrm{~h}$ at $70{ }^{\circ} \mathrm{C}$, at the end of which time TLC (petroleum ether-EtOAc 1:1) indicated completion of the reaction. The mixture was concd. under diminished pressure and then coevaporated with toluene $(3 \times 10 \mathrm{~mL})$. The residue was passed through a short silica-gel column with petroleum ether-EtOAc 3:1 as the eluent to give $7(3.1 \mathrm{~g}, 91 \%)$ as a white solid. $[\alpha]_{D}^{25}-14.4^{\circ}\left(c 1.0 \mathrm{CHCl}_{3}\right) .{ }^{1} \mathrm{H}-\mathrm{NMR}\left(300 \mathrm{MHz}, \mathrm{CDCl}_{3}\right): \delta$ 8.31-7.23 (m, 24H, Bz-H, Ar- $H), 6.05\left(\mathrm{t}, J_{3,4}=J_{4,5}=10.1 \mathrm{~Hz}, 1 \mathrm{H}, \mathrm{H}-4\right), 5.81\left(\mathrm{~d}, J_{2,3}=3.2 \mathrm{~Hz}\right.$, $\left.J_{3,4}=10.1 \mathrm{~Hz}, 1 \mathrm{H}, \mathrm{H}-3^{\prime}\right), 5.77\left(\mathrm{~d}, J_{1,2}=1.6 \mathrm{~Hz}, 1 \mathrm{H}, \mathrm{H}-1^{\prime}\right), 5.65\left(\mathrm{dd}, J_{1,2}=1.6 \mathrm{~Hz}, J_{2,3}=3.2 \mathrm{~Hz}, 1 \mathrm{H}\right.$, H-2'), $5.32\left(\mathrm{t}, J_{3,4}=J_{4,5}=9.6 \mathrm{~Hz}, 1 \mathrm{H}, \mathrm{H}-4\right), 5.13\left(\mathrm{~d}, J_{1,2}=1.6 \mathrm{~Hz}, 1 \mathrm{H}, \mathrm{H}-1^{\prime}\right), 4.73-4.69$ (m, 1H, H-3), $4.50-4.43(\mathrm{~m}, 2 \mathrm{H}), 4.27-4.20(\mathrm{~m}, 2 \mathrm{H}), 3.99-3.91(\mathrm{~m}, 2 \mathrm{H}), 3.64(\mathrm{~d}, 1 \mathrm{H}, J 9.5 \mathrm{~Hz}, \mathrm{OH}), 3.36-3.30(\mathrm{~m}$, 2H, H-6, OH), $2.17\left(\mathrm{~s}, 3 \mathrm{H}, \mathrm{COCH}_{3}\right) .{ }^{13} \mathrm{C}-\mathrm{NMR}\left(75 \mathrm{MHz}, \mathrm{CDCl}_{3}\right): \delta 171.3\left(\mathrm{COCH}_{3}\right), 166.1,165.6$, $165.5,165.4(4 \times C \mathrm{OPh}), 160.6\left(\mathrm{CNO}_{2}\right), 142.9,133.5,133.4,133.2,133.1,129.8,129.7,129.6,129.1$, 129.0, 128.8, 128.6, 128.5, 128.4, 128.2, 126.0, 116.2, 97.73, $97.70(2 \times \mathrm{C}-1), 77.2,70.4,70.3,70.1$, 69.9, 69.8, 69.3, 69.1, 66.8, 66.6, 62.8, $20.8\left(\mathrm{COCH}_{3}\right)$. HRMS for $\mathrm{C}_{48} \mathrm{H}_{47} \mathrm{~N}_{2} \mathrm{O}_{18}\left(\mathrm{M}+\mathrm{NH}_{4}\right)^{+}$939.28184. Found: 939.28088 .

4-Nitrophenyl 2,3,4,6-tetra-O-benzoyl- $\alpha$-D-mannopyranosyl-(1 $\rightarrow 6)$-[2,3,4,6-tetra-O-benzoyl- $\alpha-D-$ mannopyranosyl-(1 $\rightarrow 3)$ ]-4-O-acetyl- $\alpha$-D-mannopyranoside $(\mathbf{8})$ : Glycosylation between disaccharide acceptor 7 (2.8 g, $3 \mathrm{mmol})$ and monosaccharide donor 4 (2.4 g, $3.1 \mathrm{mmol})$ was accomplished by following the same procedure as described above for the preparation of ditrasaccharide $\mathbf{5}$. After purification, trisaccharide $8(3.9 \mathrm{~g}, 86 \%)$ was afforded as a white foamy solid. $[\alpha]_{D}^{25}-27.2^{\circ}(c 0.3$ $\mathrm{CHCl}_{3}$ ). ${ }^{1} \mathrm{H}-\mathrm{NMR}\left(300 \mathrm{MHz}, \mathrm{CDCl}_{3}\right): \delta 8.30-7.16(\mathrm{~m}, 44 \mathrm{H}, \mathrm{Bz}-H, \mathrm{Ar}-H), 6.09\left(\mathrm{t}, J_{3,4}=J_{4,5}=10.1 \mathrm{~Hz}, 2 \mathrm{H}\right.$, H-4', H-4"), 5.93 (dd, $\left.J_{2,3}=3.3 \mathrm{~Hz}, J_{3,4}=10.1 \mathrm{~Hz}, 1 \mathrm{H}, \mathrm{H}-3^{\prime}\right), 5.80$ (dd, $J_{2,3}=3.3 \mathrm{~Hz}, J_{3,4}=10.1 \mathrm{~Hz}, 1 \mathrm{H}$, H-3"), 5.63-5.51 (m, 4H, H-2', H-2", H-1', H-4), 5.42 (s, 1H, H-1"), 5.10 (d, $J_{1,2}=1.6$ Hz, 1H, H-1), $4.77-4.63(\mathrm{~m}, 4 \mathrm{H}), 4.52-4.44(\mathrm{~m}, 3 \mathrm{H}), 4.35\left(\mathrm{dd}, J_{2,3}=3.1 \mathrm{~Hz}, J_{3,4}=9.5 \mathrm{~Hz}, 1 \mathrm{H}, \mathrm{H}-3\right), 4.0(\mathrm{~d}, J=8.8 \mathrm{~Hz}$, 2H, 2H-6), 3.61 (d, $J=8.8 \mathrm{~Hz}, 1 \mathrm{H}, \mathrm{H}-6), 3.06(\mathrm{~d}, J=4.9 \mathrm{~Hz}, 1 \mathrm{H}, \mathrm{OH}), 2.27$ (s, 3H, COCH$).{ }^{13} \mathrm{C}$ NMR $(75$ $\left.\mathrm{MHz}, \mathrm{CDCl}_{3}\right): \delta 170.3\left(\mathrm{COCH}_{3}\right), 166.2,166.1,165.6(2), 165.4,165.3(2), 165.0(8 \times \mathrm{COPh}), 160.3$ $\left(\mathrm{CNO}_{2}\right), 143.0,133.6,133.4,133.3,133.0,129.8,129.75,129.7,129.6,129.56,129.50,129.1,129.0$, $128.99,128.91,128.8,128.7,128.6,128.5,128.4,128.3,128.28,128.22,128.1,125.9,116.2$, 99.6, 97.6, $97.5(3 \times \mathrm{C}-1), 79.0,77.2,70.8,70.6,70.3,69.8,69.7,69.6,69.4,69.0,67.1,67.0,66.7,63.4$, 62.8, $20.7\left(\mathrm{COCH}_{3}\right)$. HRMS for $\mathrm{C}_{82} \mathrm{H}_{69} \mathrm{NO}_{27} \mathrm{Na}(\mathrm{M}+\mathrm{Na})^{+}$1522.39508. Found: 1522.39463.

4-Nitrophenyl 2,3,4,6-tetra-O-benzoyl- $\alpha$-D-mannopyranosyl-(1 $\rightarrow 6)$-[2,3,4,6-tetra-O-benzoyl- $\alpha$-Dmannopyranosyl-(1 $\rightarrow 3)$ ]-2,4-di-O-acetyl- $\alpha$-D-mannopyranoside (9): To a solution of 8 (105 $\mathrm{mg}$, $0.07 \mathrm{mmol})$ in pyridine $(2 \mathrm{~mL})$ was added $\mathrm{Ac}_{2} \mathrm{O}(1 \mathrm{~mL})$. The reaction mixture was stirred at $\mathrm{rt}$ for $12 \mathrm{~h}$, at the end of which time TLC (petroleum ether-EtOAc 2:1) indicated that the reaction was complete. The reaction mixture was concentrated, and then the residue was purified by flash column chromatography on a silica gel column (petroleum ether-EtOAc 3:1) to give compound 9 (102 mg, 
95\%) as a yellow syrup. ${ }^{1} \mathrm{H}-\mathrm{NMR}\left(300 \mathrm{MHz}, \mathrm{CDCl}_{3}\right): \delta 8.30-7.16(\mathrm{~m}, 44 \mathrm{H}, \mathrm{Bz}-H, \mathrm{Ar}-H), 6.16,6.11(2 \mathrm{t}$, $\left.J_{3,4}=J_{4,5}=9.9 \mathrm{~Hz}, 2 \mathrm{H}, \mathrm{H}-4{ }^{\prime}, \mathrm{H}_{-} 4^{\prime \prime}\right), 5.83$ (dd, $\left.J_{2,3}=3.3 \mathrm{~Hz}, J_{3,4}=9.9 \mathrm{~Hz}, 1 \mathrm{H}, \mathrm{H}-3{ }^{\prime}\right), 5.80$ (dd, $J_{2,3}=3.3 \mathrm{~Hz}$, $\left.J_{3,4}=9.9 \mathrm{~Hz}, 1 \mathrm{H}, \mathrm{H}-3 "\right), 5.70$ (d, $\left.J_{1,2}=1.6 \mathrm{~Hz}, 1 \mathrm{H}, \mathrm{H}-1^{\prime}\right), 5.65$ (dd, $\left.J_{1,2}=1.6 \mathrm{~Hz}, J_{2,3}=3.3 \mathrm{~Hz}, 1 \mathrm{H}, \mathrm{H}-2^{\prime}\right)$, $5.58\left(\mathrm{dd}, J_{1,2}=1.6 \mathrm{~Hz}, J_{2,3}=3.3 \mathrm{~Hz}, 1 \mathrm{H}, \mathrm{H}-2 "\right) 5.53$ (m, 2H, H-2", H-4), 5.44 (d, $\left.J_{1,2}=1.6 \mathrm{~Hz}, 1 \mathrm{H}, \mathrm{H}-1 "\right)$, $5.07\left(\mathrm{~d}, J_{1,2}=1.5 \mathrm{~Hz}, 1 \mathrm{H}, \mathrm{H}-1\right) 4.71-4.48(\mathrm{~m}, 7 \mathrm{H}), 4.05-4.00(\mathrm{~m}, 2 \mathrm{H}), 3.61(\mathrm{~d}, J=8.8 \mathrm{~Hz}, 1 \mathrm{H}, \mathrm{H}-6)$, $2.36\left(\mathrm{~s}, 3 \mathrm{H}, \mathrm{COCH}_{3}\right), 2.28\left(\mathrm{~s}, 3 \mathrm{H}, \mathrm{COCH}_{3}\right) .{ }^{13} \mathrm{C}-\mathrm{NMR}\left(75 \mathrm{MHz}, \mathrm{CDCl}_{3}\right): \delta 170.6,170.0\left(2 \times \mathrm{COCH}_{3}\right)$, 166.2, 166.1, 165.6, 165.5, 165.4, 165.3, 165.2(2) $(8 \times C \mathrm{OPh}), 160.0\left(\mathrm{CNO}_{2}\right), 143.3,133.6,133.5$, $133.4,133.2$, 133.1, 132.9, 129.8, 129.7, 129.5, 129.2, 129.1, 129.0, 128.98, 128.95, 128.6, 128.57, 128.52, 128.4, 128.36, 128.31, 128.2, 126.0, 116.4, 99.1, 97.6, 95.7 (3 × C-1), 77.2, 74.2, 70.8, 70.7, $70.3,70.2,69.9,69.7,69.2,69.1,67.7,66.8,66.7,66.6,62.9,62.8,20.7,20.8\left(2 \times \mathrm{COCH}_{3}\right)$. HRMS for $\mathrm{C}_{84} \mathrm{H}_{71} \mathrm{NO}_{28} \mathrm{Na}(\mathrm{M}+\mathrm{Na})^{+} 1564.40562$. Found: 1564.40820 .

4-Nitrophenyl $\alpha$-D-mannopyranosyl- $(1 \rightarrow 6)$-[ $\alpha$-D-mannopyranosyl-( $1 \rightarrow 3)]-\alpha$-D-mannopyranoside $(\mathbf{1 0})$ : Compound 8 (3.2 g, $2.1 \mathrm{mmol})$ was dissolved in satd $\mathrm{NH}_{3}-\mathrm{MeOH}(120 \mathrm{~mL})$. After $120 \mathrm{~h}$ at $\mathrm{rt}$, the reaction mixture was concentrated to a total volume of about $5 \mathrm{~mL}$, then warm acetone $\left(50 \mathrm{~mL}, 50{ }^{\circ} \mathrm{C}\right)$ was added to the mixture under vigorous stirring, and a white solid precipitate from the solution, after kept at $0{ }^{\circ} \mathrm{C}$ for $24 \mathrm{~h}$, target compound 10 was collected after filtration (973 mg, 73\%) as white solid. $[\alpha]_{D}^{25}+54.2^{\circ}\left(c\right.$ 0.1 DMSO). ${ }^{1} \mathrm{H}-\mathrm{NMR}\left(300 \mathrm{MHz}, \mathrm{D}_{2} \mathrm{O}\right): \delta 8.20(\mathrm{~d}, J=9.2 \mathrm{~Hz}, 2 \mathrm{H}, \mathrm{Ar}-H), 7.20(\mathrm{~d}$, $J=9.2 \mathrm{~Hz}, 2 \mathrm{H}, \mathrm{Ar}-H), 5.69$ (s, $1 \mathrm{H}, \mathrm{H}-1), 5.13(\mathrm{~s}, 1 \mathrm{H}, H-1$ '), 4.30 (m, $1 \mathrm{H}, H-2), 4.11$ (dd, $J_{2,3}=3.2 \mathrm{~Hz}$, $\left.J_{3,4}=9.3 \mathrm{~Hz}, 1 \mathrm{H}, H-3\right), 4.04\left(\mathrm{~m}, 1 \mathrm{H}, H-2^{\prime}\right), 3.90-3.80(\mathrm{~m}, 5 \mathrm{H}), 3.78-3.74(\mathrm{~m}, 3 \mathrm{H}), 3.68(\mathrm{~m}, 2 \mathrm{H})$, 3.64-3.61 (m, 2H), 3.57-3.54 (m, 2H). ${ }^{13} \mathrm{C}-\mathrm{NMR}\left(75 \mathrm{MHz}, \mathrm{D}_{2} \mathrm{O}\right): \delta 160.6\left(\mathrm{CNO}_{2}\right), 142.4,126.1(2)$, 116.9(2) $(4 \times \mathrm{Ar}-\mathrm{C}), 102.4,98.9,97.6(3 \times \mathrm{C}-1), 77.9,73.2,72.6,71.9,70.6,70.4,70.1,69.9,69.2$, 66.8, 66.7, 65.8. 65.2, 61.0, 60.9. HRMS for $\mathrm{C}_{24} \mathrm{H}_{35} \mathrm{NO}_{18} \mathrm{Na}(\mathrm{M}+\mathrm{Na})^{+}$648.1752. Found: 648.1754.

\section{Conclusions}

In summary, a highly efficient and regioselective procedure with a simple work-up which is applicable on a large-scale has been developed for the direct synthesis of the 2,3-O-isopropylidene- $\alpha$ D-mannopyranoside derivatives from $\alpha$-D-mannopyranosides. The high selectivity, high isolated yields, and widely application of the reaction highlight the usefulness of the method as a practical orthogonal protecting strategy in carbohydrate synthesis. The synthetic utility of this novel process was demonstrated through the successfully synthesis of a 3,6-branched $\alpha$-D-mannosyl trisaccharide. It is noteworthy that overall yield of the whole synthesis is $50.4 \%$ from the corresponding mannoside, and the procedure is suitable for large scale preparation of the target trisaccharide.

\section{Supplementary Materials}

Supplementary materials can be accessed at: http://www.mdpi.com/1420-3049/19/5/6683/s1.

\section{Acknowledgments}

This work was partially supported by the NSFC (21172257), the National 863 Program (2011AA10A206) and the National S\&T Pillar Program (2012BAK25B03-01, 2010CB126105) of China. 


\section{Author Contributions}

Main text paragraph Rui Jiang was in charge of all the synthesis experiments and wrote the manuscript; Guanghui Zong provided help in the synthesis experiments; ${ }^{1} \mathrm{H}$ and ${ }^{13} \mathrm{C}-\mathrm{NMR}$ spectra were texted by Xiaomei Liang; Jianjun Zhang provided guidance and suggestions for all the experiments and he also provided proper suggestions when wrote and revised the manuscript; Shuhui Jin and Daoquan Wang provided guidance and suggestions in the synthesis experiments.

\section{Conflicts of Interest}

The authors declare no conflict of interest.

\section{References}

1. Wang, C.C.; Lee, J.C.; Luo, S.Y.; Kulkarni, S.S.; Huang, Y.W.; Lee, C.C.; Chang, K.L.; Hung, S.C. Regioselective one-pot protection of carbohydrates. Nature 2007, 446, 896-899.

2. Seeberger, P.H.; Werz, D.B. Synthesis and medical applications of oligosaccharides. Nature 2007, 446, 1046-1051.

3. Guo, J.; Ye, X.S. Protecting groups in carbohydrate chemistry: Influence on stereoselectivity of glycosylations. Molecules 2010, 15, 7235-7265.

4. Bertozzi, C.R.; Kiessling, L.L. Chemical glycobiology. Science 2001, 291, 2357-2364.

5. Liu, C.; Skogman, F.; Cai, Y.; Lowary, T.L. Synthesis of the 'primer-adaptor' trisaccharide moiety of Escherichia coli O8, O9, and O9a lipopolysaccharide. Carbohydr. Res. 2007, 342, 2818-2825.

6. Gigg, J.; Gigg, R.; Payne, S.; Conant, R. Synthesis of propyl 4-O-(3,6-di-O-methyl- $\beta$-Dglucopyranosyl)-2,3-di-O-methyl- $\alpha$-D-rhamnopyranoside. Carbohydr. Res. 1985, 141, 91-97.

7. Chung, S.K.; Moon, S.H. Synthesis and biological activities of (4,6-di-O-phosphonato- $\beta$-Dmannopyranosyl)-methylphosphonate as an analogue of 11-myo-inositol 1,4,5-trisphosphate. Carbohydr. Res. 1994, 260, 39-50.

8. Evans, M.E.; Parrish, F.W. Monomolar acetalations of methyl $\alpha$-D-mannosides-synthesis of methyl $\alpha$-D-talopyranoside. Carbohydr. Res. 1977, 54, 105-114.

9. Fleet, G.W.; Smith, P.W. Enantiospecific syntheses of deoxymannojirimycin, fagomine and 2r, 5r-dihydroxymethyl-3r, 4r-dihydroxypyrrolidine from D-glucose. Tetrahedron Lett. 1985, 26, 1469-1472.

10. Yadav, J.; Chander, M.C.; Reddy, K.K. Stereoselective synthesis of 10(S), 11(R), 12(R)trihydroxyeicosa-5(Z), 8(Z), 14(Z)-trienoic acid from d-mannose. Tetrahedron Lett. 1992, 33, 135-138.

11. Manna, S.; Viala, J.; Yadagiri, P.; Falck, J. Synthesis of 12(S), 20-, 12(S), 19(R)-, and 12(S), 19(S)-dihydroxyeicosa-cis-5,8,14-trans-10-tetraenoic acids, metabolites of 12(S)-hete. Tetrahedron Lett. 1986, 27, 2679-2682.

12. Vijayasaradhi, S.; Singh, J.; Aidhen, I.S. An Efficient, Selective Hydrolysis of Terminal Isopropylidene Acetal Protection by $\mathrm{Zn}\left(\mathrm{NO}_{3}\right)_{2} 6 \mathrm{H}_{2} \mathrm{O}$ in Acetonitrile. Synlett 2000, 1, 110-112. 
13. Swamy, N.R.; Venkateswarlu, Y. A mild and efficient method for chemoselective deprotection of acetonides by bismuth (III) trichloride. Tetrahedron Lett. 2002, 43, 7549-7552.

14. Ki, H.P.; Yong, J.Y.; Sang, G.L. Efficient cleavage of terminal acetonide group: Chirospecific synthesis of 2, 5-dideoxy-2, 5-imino-D-mannitol. Tetrahedron Lett. 1994, 35, 9737-9740.

15. Kim, K.S.; Song, Y.H.; Lee, B.H.; Hahn, C.S. Efficient and selective cleavage of acetals and ketals using ferric chloride adsorbed on silica gel. J. Org. Chem. 1986, 51, 404-407.

16. Mahender, G.; Ramu, R.; Ramesh, C.; Das, B. A simple and facile chemo-and regioselective deprotection of acetonides using silica supported sodium hydrogen sulfate as a heterogeneous catalyst. Chem. Lett. 2003, 8, 734-735.

17. Agarwal, A.; Vankar, Y.D. Selective deprotection of terminal isopropylidene acetals and trityl ethers using $\mathrm{HClO}_{4}$ supported on silica gel. Carbohydr. Res. 2005, 340, 1661-1667.

18. Fauré, R.; Shiao, T.C.; Damerval, S.; Roy, R. Practical synthesis of valuable d-rhamnoside building blocks for oligosaccharide synthesis. Tetrahedron Lett. 2007, 48, 2385-2388.

19. Zong, G.; Yu, N.; Xu, Y.; Zhang, J.; Wang, D.; Liang, X. Synthesis of a mannose hexasaccharide related to the cell wall mannan of candida dubliniensis and trychophyton mentagrophytes. Synthesis 2010, 10, 1666-1672.

20. Cheng, L.; Chen, Q.; Liu, J.; Du, Y. Synthesis of a fluorescence-labeled K30 antigen repeating unit using click chemistry. Carbohydr. Res. 2007, 342, 975-981.

21. Zegelaar-Jaarsveld, K.; Duynstee, H.I.; van der Marel, G.A.; van Boom, J.H. Iodonium ion-assisted synthesis of tetrameric fragments corresponding to the cell wall phenolic glycolipids of Mycobacterium kansasii serovars II and IV. Tetrahedron 1996, 52, 3575-3592.

22. Lemanski, G.; Ziegler, T. Intramolecular mannosylations of glucose derivatives via prearranged glycosides. Helv. Chim. Acta 2000, 83, 2655-2675.

23. Rana, S.S.; Barlow, J.J.; Matta, K.L. Synthetic studies in carbohydrates. Part XVIII. Synthesis of p-nitrophenyl 6-O-(2-acetamido-2-deoxy- $\beta$-D-glucopyranosyl)- $\alpha$-D-mannopyranoside. Carbohydr. Res. 1981, 96, 79-85.

24. Sparrow, L.G.; Lawrence, M.C.; Gorman, J.J.; Strike, P.M.; Robinson, C.P.; McKern, N.M.; Ward, C.W. N-linked glycans of the human insulin receptor and their distribution over the crystal structure. Proteins: Struct. Funct. Bioinform. 2008, 71, 426-439.

25. De Leoz, M.L.A.; Young, L.J.T.; An, H.J.; Kronewitter, S.R.; Kim, J.; Miyamoto, S.; Borowsky, A.D.; Chew, H.K.; Lebrilla, C.B. High-mannose glycans are elevated during breast cancer progression. Mol. Cell. Proteomics 2011, 10, 1-9.

26. Moremen, K.W.; Tiemeyer, M.; Nairn, A.V. Vertebrate protein glycosylation: Diversity, synthesis and function. Nat. Rev. Mol. Cell Bio. 2012, 13, 448-462.

27. Nairn, A.V.; Aoki, K.; dela Rosa, M.; Porterfield, M.; Lim, J.M.; Kulik, M.; Pierce, J.M.; Wells, L.; Dalton, S.; Tiemeyer, M.; et al. Regulation of glycan structures in murine embryonic stem cells. combined transcript profiling of glycan-related genes and glycan structural analysis. J. Biol. Chem. 2012, 287, 37835-37856.

28. Zhang, J.J.; Kong, F.Z. Efficient and practical syntheses of mannose tri-, tetra-, penta-, hexa-, hepta-, and octasaccharides existing in N-glycans. Tetrahedron: Asymmetry 2002, 13, 243-252.

29. Zhang, J.J.; Kong, F.Z. A facile large scale synthesis of the core mannose pentasaccharide of Nlinked glycoprotein and its isomer. Acta Chim. Sinica 2002, 1, 150-156. 
30. Mikkelsen, L.M.; Krintel, S.L.; Jiménez-Barbero, J.; Skrydstrup, T. Application of the anomeric samarium route for the convergent synthesis of the C-linked trisaccharide $\alpha$-D-Man- $(1 \rightarrow 3)-[\alpha-D-$ man- $(1 \rightarrow 6)$ ]-D-man and the disaccharides $\alpha$-D-man-( $1 \rightarrow 3)$-D-man and $\alpha$-D-man-( $1 \rightarrow 6)$-D-man. J. Org. Chem. 2002, 67, 6297-6308.

31. Liu, Y.; Chen, G. Chemical synthesis of N-linked glycans carrying both mannose-6-phosphate and glcnac-mannose-6-phosphate motifs. J. Org. Chem. 2011, 76, 8682-8689.

32. Ogawa, T.; Katano, K.; Matsui, M. Regio- and stereo-controlled synthesis of core oligosaccharides of glycopeptides. Carbohydr. Res. 1978, 64, C3-C9.

33. Winnik, F.M.; Brisson, J.R.; Carver, J.P.; Krepinsky, J.J. Syntheses of model oligosaccharides of biological significance. Synthesis of methyl 3,6-di-O-( $\alpha$-D-mannopyranosyl)- $\alpha$-D-mannopyranoside and the corresponding mannobiosides. Carbohydr. Res. 1982, 103, 15-28.

34. Kaur, K.J.; Alton, G.; Hindsgaul, O. Use of N-acetylglucosaminyltransferases I and II in the preparative synthesis of oligosaccharides. Carbohydr. Res. 1991, 210, 145-153.

35. Kaur, K.J.; Hindsgaul, O. A simple synthesis of octyl 3,6-di-O-( $\alpha$-D-mannopyranosyl)- $\beta$-Dmannopyranoside and its use as an acceptor for the assay of $\mathrm{N}$-acetylglucosaminyltransferase-I activity. Glycoconjugate J. 1991, 8, 90-94.

36. Oscarson, S.; Tiden, A.K. Synthesis of the octyl and tetradecyl glycosides of 3,6-di- $O$ - $\alpha$-Dmannopyranose and of 3,4-di- $O$ - $\alpha$-D-mannopyranosyl- $\alpha$-D-mannopyranose. A new way for 2,4-di-O-protection of mannopyranosides. Carbohydr. Res. 1993, 247, 323-328.

37. Figueroa-Perez, S.; Verez-Bencomo, V.J. Synthesis of neoglycolipids containing oligosaccharides based on 3,6-branched- $\alpha$-D-mannopyranosides as the carbohydrate moieties. Carbohydr. Chem. 1998, 17, 851-868.

38. Tanaka, H.; Nishida, Y.; Kobayashi, K. A facile synthesis of a glycoconjugate cationic polymer carrying the 3,6-branched $\alpha$-D-mannosyl trisaccharide cluster. J. Carbohydr. Chem. 2000, 19, 413-418.

39. Ratner, D.M.; Plante, O.J.; Seeberger, P.H. A linear synthesis of branched high-mannose oligosaccharides from the HIV-1 viral surface envelope glycoprotein gp120. Eur. J. Org. Chem. 2002, 5, 826-833.

40. Abronina, P.I.; Backinowsky, L.V.; Grachev, A.A.; Sedinkin, S.L.; Malysheva, N.N. An easy access to a 3,6-branched mannopentaoside bearing one terminal [1-13C]-labeled D-mannopyranose residue. Russ. Chem. Bull. 2005, 54, 1287-1293.

41. Mukhopadhyay, B.; Maurer, S.V.; Rudolph, N.; van Well, R.M.; Russell, D.A.; Field, R.A. From solution phase to "on-column" chemistry: Trichloroacetimidate-based glycosylation promoted by perchloric acid-silica. J. Org. Chem. 2005, 70, 9059-9062.

42. Arnarp, J.; Lonngren, J. Synthesis of 3,6-di-O-( $\alpha$-D-mannopyranosyl)-D-mannose. Acta Chem. Scand. Ser. B 1978, B32, 696.

Sample Availability: Samples of the compounds 1a, $\mathbf{2 a}, \mathbf{3 a}-\mathbf{g}, \mathbf{4 - 8}, \mathbf{1 0}$ are available from the authors.

(C) 2014 by the authors; licensee MDPI, Basel, Switzerland. This article is an open access article distributed under the terms and conditions of the Creative Commons Attribution license (http://creativecommons.org/licenses/by/3.0/). 